\title{
La recherche du consentement : enjeux et méthode dans une enquête qualitative auprès de personnes en fragilité psychique
}

\section{Sara Painter}

\section{CpenEdition}

Journals

Édition électronique

URL : https://journals.openedition.org/cdg/5587

DOI : $10.4000 /$ cdg. 5587

ISSN : 2107-7266

Éditeur

UMR 245 - CESSMA

Référence électronique

Sara Painter, « La recherche du consentement : enjeux et méthode dans une enquête qualitative auprès de personnes en fragilité psychique », Carnets de géographes [En ligne], 14 | 2020, mis en ligne le 15 décembre 2020, consulté le 21 mai 2021. URL : http://journals.openedition.org/cdg/5587 ; DOI : https://doi.org/10.4000/cdg.5587

Ce document a été généré automatiquement le 21 mai 2021.

\section{cc) (†)}

La revue Carnets de géographes est mise à disposition selon les termes de la Licence Creative Commons Attribution - Pas d'Utilisation Commerciale - Pas de Modification 4.0 International. 


\title{
La recherche du consentement : enjeux et méthode dans une enquête qualitative auprès de personnes en fragilité psychique
}

\author{
Sara Painter
}

1 L'article est issu d'une enquête qualitative auprès de personnes en fragilité psychique vieillissantes, vivant dans une petite ville française et membres d'une association d'entraide entre pairs appelée un GEM (Groupe d'Entraide Mutuelle). L'objectif de cette recherche était d'analyser leurs pratiques spatiales quotidiennes afin 1) d'identifier les facteurs contraignant et/ou favorisant leur inclusion dans la ville et 2) d'étudier en quoi le GEM participait à la construction des géographies quotidiennes et à l'inclusion dans la ville de ces personnes. Cette recherche a la particularité d'avoir été menée dans le cadre d'une cotutelle de thèse franco-québécoise ${ }^{1}$ en géographie sociale et en études urbaines : elle porte sur un terrain français mais la méthodologie d'enquête a été soumise aux exigences du comité d'éthique d'une université québécoise. L'article vise à partager cette expérience formatrice, qui oblige le chercheur à s'interroger sur l'impact de ses travaux et de sa présence sur le terrain de recherche.

2 L'article s'intéresse à la manière dont les exigences éthiques de ce comité ont influencé les choix méthodologiques et la réalisation de l'enquête de terrain. On verra notamment en quoi la démarche méthodologique a pu favoriser l'exercice du consentement libre et éclairé des participants à la recherche. Des éléments de cadrage sont présentés dans la première partie. La deuxième partie traite de la consignation du consentement, qui doit être adaptée aux réalités du terrain. La troisième partie souligne en quoi l'observation participante a favorisé l'exercice d'un consentement libre et éclairé des participants. 


\section{Contexte de la recherche}

\section{Une recherche qualitative auprès de personnes en fragilité psychique vieillissantes}

3 Cette recherche porte sur une population parfois qualifiée dans les études scientifiques de « vulnérable » du fait des multiples exclusions dont elle fait l'objet. Les personnes en fragilité psychique vieillissent plus précocement et moins bien que le reste de la population, avec davantage de problèmes de santé physique, une qualité de vie moindre et des ressources financières limitées (Dallaire et al., 2010). Confrontées à une double discrimination liée à l'âge et aux problèmes de santé mentale, ces personnes ont peu l'occasion d'interagir avec d'autres et de pratiquer des loisirs (Nour et al., 2010).

Peu d'études portent sur les personnes en fragilité psychique qui vieillissent. Lorsqu'elles existent, la plupart reposent sur des enquêtes quantitatives, surtout dans le domaine de la psychologie (Cummings, Kropf, 2011 ; Futeran, Draper, 2012 ; RaucherChéné et al., 2015). Cela peut en partie s'expliquer par le fait que les chercheurs expriment parfois des réticences à intégrer le point de vue de ces personnes, estimant du fait de leurs problèmes de santé mentale qu'elles ne seraient pas capables de rendre compte «objectivement " de leur fonctionnement et de leurs besoins (Meester et al., 2010). Plusieurs chercheurs rejettent cette idée et soulignent au contraire l'intérêt de mener davantage de recherches qualitatives afin de mieux comprendre les expériences vécues par cette population. Ces auteurs insistent sur la nécessité de prendre davantage en compte le discours porté directement par ces personnes sur les expériences qu'elles vivent, en vue de mieux comprendre leur quotidien et la manière dont elles font face aux difficultés (Martinsson et al., 2012 ; Tryssenaar et al., 2009).

Dans une approche portant sur les rapports des individus à la ville, il parait essentiel de s'intéresser au vécu des personnes, à ce qu'elles disent et pensent de leurs pratiques spatiales. C'est pourquoi j'ai voulu mettre en œuvre une méthodologie offrant aux participants la possibilité de s'exprimer, sans préjuger de leur capacité à parler de leur quotidien.

\section{La demande de certification éthique}

Les chercheurs ayant mené des études qualitatives auprès de personnes en fragilité psychique soulignent l'importance des considérations éthiques lors du recueil de leur parole (Parr, 2011). Ces personnes peuvent avoir des difficultés relationnelles (Lovell et al., 2009), des problèmes de mémoire et de concentration, etc. Pour certaines d'entre elles, la situation d'entretien avec un chercheur peut être stressante. Toutes ces particularités font qu'il semblait inadéquat de me limiter à un entretien semi-dirigé, sans réflexion sur la manière d'amener la situation d'entrevue auprès de ces personnes.

7 En même temps, les personnes en fragilité psychique constituent une population hétérogène (Parr, 2011). Il est donc difficile d'anticiper leurs besoins. Et aucune règle en matière d'éthique de la recherche ne peut être appliquée mécaniquement, quels que soient les personnes sollicitées ou le contexte.

8 J'ai pourtant dû présenter une méthodologie détaillée en amont de l'enquête, en raison de mon inscription en cotutelle de thèse avec une université du Québec, bien que le 
terrain d'étude soit situé en France. Dans les universités canadiennes, tout projet de recherche doit en effet être approuvé par un comité d'éthique de la recherche (CER) ${ }^{2}$ avant d'être mis en œuvre, quels que soient la discipline ou le domaine de recherche, ce qui n'est pas le cas en France. La demande de certification d'éthique se présentait sous la forme d'un dossier dans lequel je devais entre autres expliciter mes choix méthodologiques au regard de l'Énoncé de politique des trois Conseils (EPTC, 2014), document de référence posant les principes directeurs à respecter et qui comporte notamment un chapitre entier consacré aux recherches qualitatives.

L'obtention du consentement est un principe-clef de l'EPTC (2014). Celui-ci doit être volontaire : la personne sollicitée doit pouvoir prendre sa décision sans être soumise à de la manipulation ou à une quelconque obligation. Elle doit aussi se sentir libre de retirer son consentement à n'importe quel moment. Le consentement doit être éclairé, c'est-à-dire que les participants doivent disposer de «tous les renseignements pertinents leur permettant de prendre une décision éclairée " (EPTC, 2014: 30). L'information doit donc être transmise dans un langage adapté. Cette exigence du consentement libre et éclairé peut se révéler problématique lorsqu'il s'agit de personnes dont la capacité à consentir est sujette à caution.

Une large part de la demande de certification éthique consiste à expliciter les moyens mis en œuvre pour obtenir le consentement libre et éclairé des participants. Cela fera l'objet de cet article, qui revient sur la manière dont ces considérations éthiques ont été appliquées dans le cadre d'une recherche qualitative auprès de personnes en fragilité psychique vieillissantes.

\section{L'enquête et les participants}

11 L'enquête de terrain s'est déroulée en 2016-2017 auprès de 7 hommes et 7 femmes en fragilité psychique âgés de 50 à 70 ans, vivant dans une petite ville de 10000 habitants. La plupart ne travaillaient pas ou plus depuis longtemps. Tous avaient des problèmes de santé limitant leur capacité à se déplacer. Onze avaient un diagnostic de schizophrénie, de bipolarité ou de dépression. Les autres n'avaient pas de diagnostic ou n'avaient pas voulu révéler cette information. Six ont mentionné souffrir ou avoir souffert d'alcoolisme. Ces personnes étaient assez autonomes pour vivre en logement ordinaire.

12 Les participants ont été recrutés au sein d'un Groupe d'entraide mutuelle (GEM), une association d'entraide entre pairs définie comme «des lieux conviviaux, où des personnes peuvent s'entraider, organiser ensemble des activités visant tant au développement personnel qu'à créer des liens avec la communauté environnante " (circulaire DGAS/3B nº 2005-418 du 29 août 2005).

13 L'objectif de la méthodologie était d'accéder au quotidien de ces personnes, dans le respect des exigences éthiques de l'EPTC (2014). En plus d'une observation participante de plusieurs mois dans ce GEM, les participants ont été rencontrés à deux reprises pour des entretiens semi-directifs. 


\section{Comment consigner le consentement ?}

lettre décrivant le projet de recherche. Ce GEM était composé d'une cinquantaine d'adhérents. Il paraissait difficile de demander à chacun de signer un formulaire de consentement individuel à ma présence en tant qu'observatrice, d'autant plus que seuls quelques-uns allaient être sollicités pour des entretiens. Ce sont en fait les adhérents qui ont proposé une solution. Mon projet avait été d'abord examiné par les membres du Conseil d'Administration du GEM, composé d'adhérents élus. Ceux-ci posaient une condition : que je présente mon projet et ma demande d'observation participante lors d'une réunion rassemblant les adhérents du GEM pour qu'ils puissent voter pour ou contre. En cas de refus, ma demande aurait été rejetée. Cette méthode correspondait en fait à la manière dont ce GEM fonctionne : toutes les décisions importantes doivent être validées par le vote des adhérents. J'ai donc pu m'appuyer sur ce fonctionnement pour obtenir le consentement collectif des adhérents à ma présence en tant qu'observatrice participante. Cette méthode est apparue aux yeux du CER comme « inhabituelle » mais appropriée au regard du contexte. Lors du vote, le « oui » l'a emporté à l'unanimité.

Le CER m'a proposé d'ajouter certaines précautions. La première concernait le cas où une nouvelle personne venait à fréquenter le GEM après ce vote. Il était alors prévu que je lui explique de la même manière qu'aux autres adhérents mon projet de thèse. En cas de réticences de la personne face au projet, il était prévu que je ne collecterais pas d'informations sur elle dans mon carnet d'observation. Si un adhérent éprouvait des réticences vis-à-vis du projet même après avoir consenti collectivement lors de la réunion, je prévoyais aussi de ne pas l'intégrer dans mes observations. Si un adhérent demandait à se retirer du projet en cours, il était prévu que toutes les informations le concernant seraient ignorées dans l'analyse. Ce cas de figure n'est cependant pas apparu lors de l'enquête.

\section{Le consentement individuel aux entretiens}

17 Après plusieurs semaines d'observation, il était prévu que je mène des entretiens auprès d'une quinzaine d'adhérents. La méthode de consignation du consentement a alors été plus classique. Je faisais signer un formulaire de consentement à la personne sollicitée, rédigé dans un langage accessible et précisant que les informations collectées resteraient confidentielles, sauf si la personne était en danger (par exemple si elle exprimait des idées suicidaires). Auquel cas, il était prévu que je prévienne un salarié du GEM.

Carnets de géographes, 14 | 2020 

entretiens, quel que soit leur niveau d'autonomie ou leur statut légal. Ce choix se justifie au regard du fonctionnement des GEM, qui promeuvent la responsabilisation des personnes adhérentes. L'arrêté du 13 juillet 2011 stipule que "les adhérents du GEM participent de plein droit avec voix délibérative aux assemblées générales, qui doivent permettre de déterminer les grandes orientations du groupe d'entraide et constituer des temps d'échanges et d'information pour tous les adhérents. À ce titre, ils élisent les membres du conseil d'administration. Ceux qui sont élus administrateurs participent aux séances du conseil avec voix délibérative ». Chaque personne au GEM peut faire entendre sa voix, c'est un droit fondamental. Il aurait ainsi été très inapproprié et contre-productif de considérer le consentement personnel de certains adhérents comme insuffisant et d'exiger par exemple le consentement de leur tuteur dans le cas des majeurs protégés. Le CER a approuvé cet argument.

Ajoutons que cette recherche a été classée par le CER comme "à risque minimal», signifiant que «la probabilité et l'ampleur des préjudices éventuels découlant de la participation à la recherche ne sont pas plus grandes que celles des préjudices inhérents aux aspects de la vie quotidienne du participant qui sont associés au projet de recherche » (EPTC, 2014 : 231). L'EPTC rappelle que la notion de vulnérabilité rattachée à une population spécifique peut varier selon les recherches: "des personnes ne devraient pas automatiquement être considérées comme vulnérables uniquement en raison de suppositions quant à la vulnérabilité du groupe auquel elles appartiennent. Il faut considérer leur situation particulière dans le contexte du projet de recherche proposé. Les CER et les chercheurs doivent examiner soigneusement le lien entre la situation des personnes et des groupes qui seraient recrutés et la question de recherche en cause. Ils ne devraient pas présumer que cette situation doive automatiquement entraîner l'inclusion ou l'exclusion de personnes ou de groupes en tant que participants » (EPTC, $2014: 59)$.

Ma présence prolongée sur le terrain a favorisé l'exercice du consentement libre et éclairé des participants, en amont des entretiens mais aussi après. Plusieurs participants ont en effet complété ou modifié des propos qu'ils avaient tenus lors de l'entrevue, à propos desquels ils n'étaient pas satisfaits. Un participant était notamment inquiet de s'être trop confié lors de l'entrevue. Quelques jours après l'entretien, lors d'une après-midi passée au GEM, il a pu venir me voir et me demander de ne pas tenir compte de certains de ses propos. J'ai bien entendu accepté sa demande. Mais ce participant a quand même eu besoin de me reparler de ce sujet à plusieurs reprises, pour se rassurer et vérifier que je n'avais pas oublié sa demande. Être présente plusieurs semaines après la fin des entretiens m'a permis de le rassurer lorsqu'il en avait besoin.

\section{Comment garantir le consentement libre et éclairé ?}

\section{L'importance d'une information accessible}

21 L'accès à l'information est un autre principe essentiel du consentement. Dans le GEM étudié, tous les adhérents n'avaient pas les mêmes capacités à comprendre et à assimiler des informations. C'est pourquoi il était essentiel d'expliquer le projet de recherche dans un langage accessible à tous. Certains adhérents ont vite compris le 
sens de ma démarche. Mais globalement, il m'a fallu prendre le temps d'expliquer et de réexpliquer régulièrement le but de l'enquête et la façon dont les entretiens allaient se dérouler. Au bout de quelques temps, certains adhérents m'ont accompagnée dans cette tâche, en expliquant eux-mêmes le projet de recherche aux adhérents qui n'avaient pas bien compris ou qui avaient oublié. Beaucoup avaient des problèmes de mémoire, d'où l'importance de répéter les informations.

22 J'ai pu constater l'intérêt de multiplier les supports d'information. Certains adhérents étaient plus sensibles à l'écrit, d'autres à l'oral. Il était aussi important de ne pas être trop formel dans la présentation de l'information. J'avais d'abord laissé une feuille décrivant la recherche dans la salle commune du GEM mais je me suis vite aperçue que celle-ci retenait très peu l'attention des adhérents. J'ai donc décidé d'utiliser tous les supports à ma disposition. En plus des conversations informelles avec les adhérents, j'ai pu régulièrement rappeler la raison de ma présence lors des réunions des adhérents où je prenais la parole pour parler des avancées de l'enquête ou encore dans le journal mensuel du GEM.

Figure 1. Présentation de l'enquête aux adhérents du GEM

\section{L vie the Gem}

Le retour de SARA. Bonjour! Beaucoup d'entre vous me connaissent déjà. Mais pour celles et ceux que je n'ai pas encore croisés, voilà, je me présente. Je m'appelle Sara et je suis étudiante au doctorat, en géographie. Mon sujet d'étude porte sur le quotidien des adhérents du GEM âgés d'environ $\mathbf{5 0}$ ans et plus. Pour être plus précise, je m'intéresse aux lieux du quotidien et à la manière dont les adhérents s'y sentent. Quels sont les endroits où ils sentent bien ou pas? Inclus ou pas ? Y a-t-il endroits où ils vivent de l'exclusion ? Pour faire ce travail, j'ai besoin de passer du temps avec vous, de discuter, de mieux vous connaître. C'est pour ça que je viendrai chaque jour au GEM jusqu'à avril. Comme l'été dernier, j'aimerais faire des entretiens avec les adhérents intéressés. Je vous en parlerai à la réunion des adhérents, le 10 février. N'hésitez pas à venir me voir si vous avez des questions !

Source : journal du GEM

Pouvoir passer du temps au GEM s'est avéré utile pour que les adhérents puissent bien prendre connaissance du projet de recherche. Certains ont eu besoin de plusieurs semaines de réflexion avant d'accepter de participer à un entretien. Il leur paraissait en effet étonnant, voire incompréhensible, que je m'intéresse à leur quotidien, que beaucoup jugeaient peu digne d'intérêt. Par ailleurs, très peu d'entre eux connaissaient la géographie sociale, si bien que mon travail de recherche pouvait leur sembler assez abstrait. Peut-être en aurait été-t-il autrement avec une autre discipline. Les adhérents me demandaient en effet régulièrement pourquoi je ne faisais pas une thèse de sociologie ou de psychologie par exemple, vraisemblablement parce que ces disciplines leur étaient un peu plus familières que la géographie sociale. Le fait que je sois présente plusieurs jours par semaine leur a permis de me poser des questions, parfois au compte-goutte comme on le voit dans cet extrait du carnet de terrain :

$\mathrm{X}$ est un adhérent que j'ai sollicité pour un entretien. Il est intéressé par le projet mais il hésite à participer car il s'est déjà livré lors d'un recueil de témoignage l'an dernier et il a trouvé la démarche éprouvante. Pendant près de trois semaines, il me pose des questions par-ci par-là. Un jour il me demande en quoi consiste la géographie sociale. Quelques jours après, il me demande si ce qu'il dira pendant l'entretien sera VRAIMENT confidentiel. La semaine suivante il me demande ce que je ferai des informations récoltées pendant l'entretien et combien de temps durera notre échange. Il me demande aussi un texte de géographie sociale pour voir à quoi 
ça ressemble. Au bout de trois semaines, il se présente à moi et me dit qu'il a décidé

d'accepter l'entretien.

24 Cet exemple montre l'intérêt de combiner entretiens et observation participante et d'avoir choisi d'inscrire l'enquête dans la durée pour répondre aux questions des adhérents à mesure qu'elles leur venaient en tête. Sans ma disponibilité sur le terrain, cet adhérent aurait probablement refusé l'entretien.

\section{L'importance du lien de confiance}

J'ai pratiqué de l'observation ouverte. Les adhérents connaissaient mes intentions, ils savaient que je passais du temps avec eux pour mieux comprendre le fonctionnement du GEM et faire des entretiens. À mon arrivée, certains sont venus d'emblée vers moi : alors voilà, moi mes déplacements c'est ceci, cela. Lors de mon premier jour, l'un des adhérents s'est présenté à moi, mi-sérieux, mi-riant :

Alors tu vois, je suis bipolaire, je suis une personne vieillissante et je vis dans une petite ville, je suis un sujet idéal pour ta recherche!

Pour d'autres, plus réservés, il a fallu du temps pour qu'ils m'accordent leur confiance et acceptent de discuter avec moi, de manière informelle d'abord puis lors d'un entretien.

L'immersion de longue durée m'a amenée à tisser des liens avec les adhérents. Faire l'expérience sensible du terrain permet d'accéder à un pan supplémentaire de la réalité étudiée. C'est l'intérêt de l'immersion. C'est en interagissant de manière régulière et informelle avec les personnes qu'on les connaît mieux et qu'on apprend des choses sur leur quotidien.

Accepter de s'impliquer émotionnellement, c'est aussi faire preuve de sincérité auprès des participants, accepter de parler de soi, de s'ouvrir, dès lors qu'on leur demande de faire de même lors des entretiens. Cette réciprocité nourrit une relation de confiance et permet d'interagir avec les participants à partir de rapports plus horizontaux. Le fait de participer aux activités proposées (promenades, jeux de société, cours de dessin, etc.) m'a également permis de tisser plus facilement des liens avec les adhérents du GEM.

En expliquant ma volonté de mener une observation participante de longue durée au sein du GEM, le CER a attiré mon attention sur les risques d'influence indue, c'est-à-dire d'abus d'influence. L'EPTC alerte les chercheurs vis-à-vis « des éléments de confiance et de dépendance dans les relations", qui peuvent constituer "une source d'influence indue sur la personne en situation de dépendance, si l'objectif est d'amener cette dernière à participer à un projet de recherche » (2014: 29). Au moment de proposer les entretiens, il fallait que je sois attentive au moindre signe de réticence qui aurait pu laisser croire que finalement la personne ne voulait plus participer. J'avais également demandé aux salariées du GEM de me prévenir si l'un des participants n'était pas à l'aise à l'idée de faire un entretien mais n'osait pas m'en parler.

30 J'ai constaté que cette manière de procéder, parce qu'elle est souple, basée sur des rapports de confiance et qu'elle s'échelonne dans le temps, a justement permis à davantage d'adhérents de participer aux entretiens. Leur donner du temps est apparu comme une condition essentielle pour que ces personnes puissent réellement évaluer si elles souhaitaient ou non participer aux entretiens. Il fallait les rassurer sur la manière dont les choses allaient se passer, adopter une méthode souple qui n'allait pas les 
brusquer. Impossible alors de respecter ces exigences éthiques dans la limite des trois années de financement imposée par mon contrat doctoral ${ }^{3}$.

En ne prenant pas le temps de mettre à l'aise les participants potentiels et de leur faire comprendre que quel que soit le choix qu'ils fassent, cela ne les affectait en rien, la plupart risquait de se braquer. Nombre d'adhérents avaient des difficultés sur le plan des interactions sociales. Certains se sentaient mal à l'aise avec les autres, surtout avec des personnes inconnues. En ne prenant pas ces éléments en compte, le risque était que la personne se renferme et refuse catégoriquement, ou au contraire qu'elle accepte parce qu'elle se sentait obligée. Il était important aussi que la personne se sente suffisamment en confiance pour refuser. Sinon elle n'aurait en aucun cas exercé son consentement libre et éclairé. Ces adhérents auraient ainsi pu être injustement exclus de la recherche, parce que je n'aurais pas pris en compte le fait que leur capacité décisionnelle peut "varier selon [...] les circonstances entourant la décision ou le moment où le consentement est sollicité » (EPTC, $2014: 46)$.

Cette justification a été acceptée par le CER et s'est vérifiée sur le terrain, puisque certains adhérents ont accepté immédiatement les entretiens et d'autres ont eu besoin de plus de temps pour mieux me connaître et s'assurer de ma bonne foi.

\section{Conclusion}

Cet article a permis d'examiner l'influence de certaines exigences éthiques du CER sur les choix méthodologiques et la réalisation de l'enquête de terrain, en particulier au regard de la question du consentement libre et éclairé des participants. Concernant les résultats de la recherche, ceux-ci révèlent la multitude de facteurs contraignant les pratiques des enquêtés, en particulier au regard de leur vie sociale, des loisirs et du logement. Mais l'analyse montre aussi la manière dont les adhérents se sont saisis collectivement du GEM pour agir eux-mêmes sur la ville et la rendre davantage inclusive.

34 L'article montre comment l'observation participante s'est révélée être bien plus qu'un simple outil de collecte des données. En m'offrant la possibilité d'être très présente sur le terrain et de développer des liens avec les participants, cette technique d'investigation a permis de réunir les conditions pour qu'ils puissent exercer leur consentement libre et éclairé. Faire de la recherche qualitative auprès de personnes en fragilité psychique amène à rencontrer des individus pour lesquels les relations sociales peuvent parfois être compliquées. Pour autant, ces difficultés ne devraient pas les empêcher de participer à des projets de recherche. Il est aussi du devoir du chercheur de ne pas bousculer ces personnes, au seul motif que la recherche doit être réalisée rapidement. Pour Béatrice Collignon, l'enjeu est de reconnaître aux enquêtés leur statut de "personnes à part entière, existant en dehors de l'espace-temps du terrain, c'est-à-dire hors d'une relation qui les définit comme les interlocuteurs, parfois même "les cherchés", du chercheur" (2010: 76). Pour cela, le temps apparaît comme un facteur essentiel dans des projets de recherche qui visent à offrir aux personnes la possibilité de participer selon des modalités qui leur conviennent.

35 Les échanges avec le CER m'ont apporté des conseils qui ont nourri ma réflexion sur l'éthique de ma recherche. Devoir en amont justifier par écrit des éléments relatifs à la méthodologie oblige le chercheur à vraiment s'interroger sur la question du consentement, qu'il ne peut aucunement évincer. Il faut préciser que dans mon cas, le 
comité d'éthique s'est montré ouvert et compréhensif vis-à-vis des problématiques rencontrées sur le terrain par les chercheurs menant des recherches qualitatives. L'expérience s'est ainsi révélée plutôt positive. Mais cela n'est pas toujours le cas. Ces instances ne sont en effet pas toujours pleinement informées des enjeux et des particularités liés aux méthodologies qualitatives, notamment de l'ethnographie (Eysermann, 2010). On ne peut donc conclure cet article sans rappeler que les expériences des chercheurs avec les CER sont loin d'être toujours aisées, surtout lorsque ces instances éludent les particularités des méthodologies qualitatives et cherchent à faire appliquer de manière mécanique des règles édictées pour la recherche biomédicale, au détriment d'une véritable réflexion sur l'éthique de la recherche en sciences sociales (Gagnon, 2010), menant alors à une standardisation des pratiques (Larouche, 2019).

MEESTERS P. D., STEK M. L., COMIJS H. C., de HAAN L., PATTERSON T. L., EIKELENBOOM P., BEEKMAN A. T. (2010), « Social functioning among older community-dwelling patients with schizophrenia : a review », The American Journal of Geriatric Psychiatry, vol. 18, no. 10, pp. 862-878.

\section{BIBLIOGRAPHIE}

COLLIGNON, B., (2010) «L'éthique et le terrain », L'information géographique, vol. 74, no. 1, pp 63-83.

CUMMINGS S. M., KROPF N. P. (2011), « Aging with a severe mental illness : Challenges and treatments ", Journal of Gerontological Social Work, vol. 54, no. 2, pp. 175-188.

DALLAIRE B., MCCUBBIN M., PROVOST M., CARPTENTIER N., CLEMENT M. (2010), «Cheminements et situations de vie des personnes âgées présentant des troubles mentaux graves : perspectives d'intervenants psychosociaux », La Revue canadienne du vieillissement, vol. 29, no. 2, pp. 267-279.

EPTC (2014), Ethique de la recherche avec des êtres humains, Enoncé de Politique des Trois Conseils.

EYSERMANN B. (2010), « Un terrain dans une association caritative marseillaise (France) à l'épreuve de l'éthique de la recherche (Canada) », Éthique publique, vol. 12, no. 1, pp. 179-200.

FUTERAN S., DRAPER B. M. (2012), « An examination of the needs of older patients with chronic mental illness in public mental health services ", Aging \& mental health, vol. 16, no. 3, pp. 327-334.

GAGNON E. (2010), « Le comité d'éthique de la recherche, et au-delà », Revue internationale d'éthique sociétale et gouvernementale, vol. 12, no. 1, pp. 299-308.

LAROUCHE J. M. (2019), « Les sciences sociales et l'éthique en recherche en contexte canadien », Revue d'anthropologie des connaissances, vol. 13, no. 2, pp. 479-501.

LOVELL A. M., TROISOEUFS A., MORA M. (2009), « Du handicap psychique aux paradoxes de sa reconnaissance. Éléments d'un savoir ordinaire des personnes vivant avec un trouble psychique grave ", Revue française des affaires sociales, no. 1, pp. 207-229. 
MARTINSSON G., FAGERBERG I., LINDHOLM C., WIKLUND-GUSTIN L. (2012), « Struggling for existence-life situation experiences of older persons with mental disorders», International journal of qualitative studies on health and well-being, vol. 7, no. 1.

NOUR K., DALLAIRE B., REGENSTREIF A., HEBERT M., MOSCOVITZ N. (2010), « Santé mentale et vieillissement, Problèmes, répercussions et services ", in Charpentier M., Guberman N., Billette V., Lavoie J.P., Grenier A., Olazabal I., Vieillir au pluriel, Québec, PUQ, pp. 135-160.

PARR H. (2011), Mental health and social space: towards inclusionary geographies. John Wiley \& Sons.

RAUCHER-CHENE D., HOERTEL N., BERA-POTELLE C., TERRIEN S., BARRIERE S., DA RIN D., LIMOSIN F. (2015), « Mental healthcare in older adults with schizophrenia: results from 118 French public psychiatric departments ", International psychogeriatrics, vol. 27, no. 10, pp. 1749-1750.

TRYSSENAAR J., CHUI A., FINCH L. (2009), « Growing older: The lived experience of older persons with serious mental illness », Canadian Journal of Community Mental Health, vol. 22, no. 1, pp. 21-36.

\section{NOTES}

1. La thèse, intitulée L'inclusion dans la ville des personnes en fragilité psychique vieillissantes, a été dirigée par Raymonde Séchet (UMR ESO, Rennes) et Anne-Marie Séguin (VIES, INRS-UCS, Montréal). Elle a été soutenue le 20 juin 2019 à l'Université Rennes $2:$ http://eso-rennes.cnrs.fr/ fr/formation-a-la-recherche/theses-soutenues/painter-sara.html. Cette recherche a reçu le soutien financier du Conseil Régional de Bretagne, de l'INRS et du CREGES (Centre de recherche et d'expertise en gérontologie sociale).

2. Les CER sont constitués d'au moins 5 personnes dont deux chercheurs, une personne versée en éthique, une personne versée en droit et une personne membre de la société civile (EPTC, 2014).

3. En France, la plupart des financements doctoraux sont limités à trois ans. Au Québec, la durée du doctorat est de quatre ans. En l'occurrence, cette thèse a nécessité cinq années de travail.

\section{RÉSUMÉS}

L'article est tiré d'une enquête qualitative auprès de personnes en fragilité psychique vieillissantes, vivant dans une petite ville française et membres d'un Groupe d'Entraide Mutuelle (GEM). L'objectif de cette recherche était d'analyser leurs pratiques spatiales quotidiennes afin 1) d'identifier les facteurs contraignant et/ou favorisant leur inclusion dans la ville et 2) d'étudier en quoi le GEM participait à la construction des géographies quotidiennes et à l'inclusion dans la ville de ces personnes. La méthodologie a la particularité d'avoir été soumise aux exigences du comité d'éthique d'une université canadienne. L'article relate cette expérience en examinant l'influence de ces exigences éthiques sur la réalisation de l'enquête et l'obtention du consentement des participants.

The article stems from a qualitative research with psychologically fragile individuals, living in a small town and members of a peer-support group called a "GEM". The aim of the study was to 1) identify the factors that restricted and/or facilitated their inclusion in the city, and 2) analyse 
how the GEM contributed to the construction of their everyday geographies and their inclusion in the city. The study was conducted in France, but the methodology was first submitted to a Canadian ethics committee. The article recounts this experience by examining the influence of these ethical requirements on the field survey and on how the participants' consent was obtained.

\section{INDEX}

Thèmes : Carnets de terrain

Keywords : Consent, Ethics, Qualitative Research, Mental Health, Inclusion, City

Mots-clés : consentement, éthique, recherche qualitative, santé mentale, inclusion, ville

\section{AUTEUR}

\section{SARA PAINTER}

Docteure en géographie sociale et études urbaines, Laboratoire ESO, Université de Rennes 2, Equipe de recherche Vieillissements, exclusions sociales et solidarités (VIES), INRS-UCS

(Montréal, Canada).

sara.painter[at]outlook.fr 